\title{
Perspective
}

Nephrology

Published online: June 12, 2018

DOI: $10.1159 / 000490064$

\section{Can Emergency Department Visits for Adverse Drug Reactions in Dialysis Patients be Reduced?}

\author{
Simit Doshi Jay B. Wish \\ Division of Nephrology, Department of Medicine, Indiana University School of Medicine, Indianapolis, IN, USA
}

Patients with end-stage renal disease (ESRD) are admitted to the hospital about 2 times every year. More than a third of those patients who are discharged alive are readmitted within 30 days, a rate which is more than twice that of Medicare beneficiaries without kidney disease. The highest rates of rehospitalization (43\%) occur in dialysis patients aged 22 to 44 years indicating that this is a problem not restricted to the elderly. In-patient care carries substantial financial burden with about $33 \%$ of the Medicare budget for ESRD patients being spent on hospital admissions. Given that the total Medicare fee-forservice spending for beneficiaries with ESRD in 2015 rose to USD 33.9 billion, the magnitude of these expenses can be extrapolated [1].

A study examining predictors of 30-day readmission among maintenance hemodialysis patients from University of North Carolina Hospitals concluded that patients taking $\geq 10$ medications had about 1.7 times higher odds

\section{KARGER}

(c) 2018 S. Karger AG, Basel

E-Mail karger@karger.com

www.karger.com/ajn of being readmitted. Furthermore, a reduction in the number of outpatient medications resulted in about 70 $80 \%$ decrease in the likelihood of readmission within 30 days [2]. Medication-related problems (MRP) are a major contributor to the increase in in-patient care and emergency department (ED) visits in ESRD patients. A study focusing on the cause for MRPs identified inappropriate dosing (33.5\%) and adverse drug reactions (20.7\%) to be the most common problems. The rate of occurrence was about $0.45 \mathrm{MRP} /$ patient/month [3]. The number of hemodialysis patients in the United States as of December 31,2015 was approximately 450,000 [1], which would result in $>200,000$ MRPs per month nationally in this population.

Patients with ESRD have an average of 6 comorbid conditions and are prescribed about 12 medications, which require frequent dose adjustments and monitoring [4]. Factors that result in adverse medication reactions 
include $>3$ comorbid conditions, $>4$ medication changes over the past year, $>5$ medications in regimen, $>12$ medication doses per day, noncompliance, drugs requiring monitoring, and presence of diabetes [3]. A large proportion of dialysis patients have more than one of these risk factors and many have all. In a pooled analysis of MRP among hemodialysis patients, the top 2 categories identified were inadequate laboratory monitoring of medications $(23.5 \%)$ and inappropriate drug dosing (both under and over dosing; $20.4 \%$ ) [4].

In this issue of AJN, Chan et al. [5] present an analysis of the 2008-2013 National Emergency Department Sample database to compare ED visits for dialysis patients and propensity matched non-dialysis patients, with a focus on ED visits for adverse drug events (ADEs). While ED visits for ADEs decreased for both groups during the time period examined, they were over tenfold higher for dialysis than non-dialysis patients. After propensity matching, patient admission was higher in dialysis than non-dialysis patients ( 88 vs. $76 \%$ ) and dialysis was associated with 3 times the odds of in-patient mortality. Dialysis patients were more likely to be discharged to a skilled nursing facility (25.4 vs. $18.8 \%)$ and had a 1-day longer average length of stay in the hospital. Not surprisingly, the analysis demonstrated that the number of comorbidities correlated with the number of ADEs and related ED visits. Hemodialysis as a modality, female gender, and age $\geq 65$ years were additional risk factors for ED visits resulting from ADEs. While the overall incidence of ED visits decreased between 2008 and 2013, dialysis patients had 6 times higher risk compared to non-dialysis patients in adjusted analyses [5].

A striking observation in the Chan et al. [5] study was that anticoagulants contributed toward a significant percentage ( $\sim 19 \%$ ) of ADEs resulting in ED visits among dialysis patients. An increase in the number of ADEs was noted to be temporally related to the increased availability of novel oral anticoagulants (NOACs) when a rapid increase of about $36 \%$ in ED visits related to ADEs was observed in 2012 and 2013. Patients with ESRD are excluded from most trials of NOAC. In fact, the only NOAC approved for use in dialysis is apixaban following a study in 8 dialysis patients. Moreover, there are no reliable monitoring tests that can allow for dose adjustments, which may result in inappropriate dosing in dialysis patients. The alternative agent warfarin did not fare any better in an analysis showing 2.28 higher odds of 30-day readmission if the drug was part of the patient's outpatient regimen. The impact of warfarin use on increased readmission risk surpassed the risk from having a catheter as vascular access and taking $\geq 10$ medications [2]. This raises the question as to whether the use of anticoagulants should be reevaluated in ESRD patients. Atrial fibrillation is the most common indication for use, but there is good evidence to suggest that it does not confer an increased risk of stroke in patients with ESRD [6].

It is critical that we develop strategies to prevent ADEs and resulting ED visits, given the magnitude of the societal and financial burden. The electronic health record incentive program introduced in 2011 serves as a good example. An analysis of the impact of e-prescribing on ADEs in Medicare Part D beneficiaries showed that in patients with $\geq 75 \%$ of meds e-prescribed the rate of ADEs was significantly lower at 21/1,000 beneficiaries compared to $36 / 1,000$ in those with $50-74.9 \%$ e-prescriptions and about 40/1,000 in those below 25\% e-prescriptions [7]. Interventions should target the dialysis population at large, as the pill burden is almost universally high in these patients. While certain high-risk groups are identified in individual analysis, one analysis of prescribing patterns showed that there was no significant difference in the number of medications in young $(<65$ years) vs. older ( $\geq 65$ years) patients $[8]$.

Implementation of a clinical pharmacist managed program has also been shown to have a significant impact on preventing ADEs. In a 2-year pilot study of 104 hemodialysis patients receiving in-depth review of medications by a trained clinical pharmacist vs. standard of care, there was a significant reduction in the number of drugs (14\%). Additionally, there was a reduction in the number of hospitalizations ( $1.8 \pm 2.4$ vs. $3.1 \pm 3)$ [9]. In a 2014 editorial regarding the avoidance of hospital readmissions in ESRD patients, Hakim and Collins [10] emphasize the importance of prompt medication reconciliation following a patient's return to the dialysis unit, citing the reduction in hospital readmissions when a clinical pharmacist is involved [9]. Unfortunately, most dialysis facilities do not have a clinical pharmacist on staff, so the responsibility for medication reconciliation following hospital discharge falls on other professionals in the dialysis unit. This is often a nurse who does not have the medical nor pharmacologic knowledge to sort out duplicate medications, improper dosages, or inappropriate indications. It has been demonstrated that more frequent physician visits to the dialysis unit reduce the frequency of hospital readmissions [11] and it is likely that some of this effect is due to the role of physicians in medication reconciliation. Streja [12] proposes that post-hospital care of the dialysis patient, including medication reconciliation, 
should be entirely the responsibility of the nephrologist and not of the dialysis center.

It is clear that a multifaceted approach is required to reduce the problem of medication mismanagement, resulting ADEs, ED visits, and hospital admissions/readmissions among dialysis patients. The nephrologist responsible for the patient's care during a hospital admission must be vigilant to prevent the inappropriate prescription and dosing of medications, even though a hospital pharmacist might be providing some oversight. Once the patient is discharged from the hospital and a pharmacist is unlikely to be involved, the nephrologist must step up to perform prompt medication reconciliation, ideally with the patient bringing in his or her medication bottles as a "source of truth" as to which medications are actually being taken. This approach will likely have benefits to all stakeholders including the patients with improved outcomes, payers with decreased expenditures, and dialysis facilities and hospitals with decreased fiscal penalties for readmissions.

\section{Disclosure Statement}

None.

\section{References}

1 United States Renal Data System. 2017 USRDS Annual Data Report: Epidemiology of Kidney Disease in the United States. Bethesda, National Institutes of Health, National Institute of Diabetes and Digestive and Kidney Diseases, 2017.

- Flythe JE, Katsanos SL, Hu Y, Kshirsagar AV, Falk RJ, Moore CR: Predictors of 30-day hospital readmission among maintenance hemodialysis patients: a hospital's perspective. Clin J Am Soc Nephrol 2016;11:1005-1014.

3 Manley HJ, Drayer DK, Muther RS: Medication-Related Problem Type and Appearance Rate in Ambulatory Hemodialysis $\mathrm{Pa}$ tients. BMC Nephrol 2003;4:10. https://bmcnephrol.biomedcentral.com/track/ pdf/10.1186/1471-2369-4-10 (accessed May $5,2018)$.

4 Manley HJ, Cannella CA, Bailie GR, St Peter WL: Medication-related problems in ambula- tory hemodialysis patients: a pooled analysis. Am J Kidney Dis 2005;46:669-680.

5 Chan L, Saha A, Poojary P, Chauhan K, Naik $\mathrm{N}$, Coca $\mathrm{S}$, et al: National trends in emergency room visits of dialysis patients for adverse drug reactions. Am J Nephrol 2018;47:441-449.

6 Wiesholzer M, Harm F, Tomasec G, Barbieri G, Putz D, Balcke P: Incidence of stroke among chronic hemodialysis patients with nonrheumatic atrial fibrillation. Am J Nephrol 2001;21:35-39.

7 Gabriel MH, Powers C, Encinosa W, Bynum JP: E-prescribing and adverse drug events: an observational study of the medicare part D population with diabetes. Med Care 2017;55: 456-462.

-8 Manley HJ, Garvin CG, Drayer DK, Reid GM, Bender WL, Neufeld TK et al: Medication prescribing patterns in ambulatory haemodialysis patients: comparisons of USRDS to a large not-for-profit dialysis provider. Nephrol Dial Transplant 2004;19:1842-1848.

-9 Pai AB, Boyd A, Depczynski J, Chavez IM, Khan N, Manley H: Reduced drug use and hospitalization rates in patients undergoing hemodialysis who received pharmaceutical care: a 2-year, randomized, controlled study. Pharmacotherapy 2009;29:1433-1440.

10 Hakim RM, Collins AJ: Reducing avoidable hospital readmissions in ESRD: a shared responsibility. J Am Soc Nephrol 2014;25:18851895.

11 Erickson KF, Winkelmayer WC, Chertow GM, Bhattacharya J: Physician visits and 30day hospital readmissions in patients receiving hemodialysis. J Am Soc Nephrol 2014;25: 2079-2087.

12 Streja E. Hospital readmission for the dialysis patient: who is (not) responsible? Kidney Int 2017;92:788-790. 